\title{
A Parameterized Partial Element Equivalent Circuit Method for Sensitivity Analysis of Multiport Systems
}

\author{
Luca De Camillis, Francesco Ferranti, Member IEEE, Giulio Antonini, Senior Member IEEE, \\ Dries Vande Ginste, Member IEEE, Daniël De Zutter, Fellow IEEE
}

\begin{abstract}
This paper presents a new technique to perform parameterized sensitivity analyses of systems that depend on multiple design parameters, such as layout and substrate features. It uses the electromagnetic method called Partial Element Equivalent Circuit method to compute state space matrices at a set of design space points. These electromagnetic matrices are interpolated as function of the design parameters. The proposed interpolation scheme allows to compute the derivatives of the matrices, which are needed to perform the sensitivity analysis. An extensive study of the required stability and passivity properties of the system involved in the parameterized sensitivity analysis is presented in this work. Pertinent numerical results demonstrate the robustness, accuracy and efficiency of the proposed methodology.
\end{abstract}

Index Terms-Sensitivity analysis, transient analysis, parameterized Partial Element Equivalent Circuit (PEEC), multiport system

\section{INTRODUCTION}

$\mathbf{T}$ HE DESIGN process of an electromagnetic (EM) system aims at obtaining the optimal values of the design variables for which the system responses satisfy the design specifications. This process is usually carried out on computers through EM simulations. Optimal values of the design variables are usually determined using optimization algorithms (optimizers). These algorithms drive the EM simulators to obtain the responses and their sensitivities in each optimization iteration. Traditional EM-based optimization techniques estimate the responses sensitivities required by the optimizer through a finite-difference approach (FDA), which invokes the EM simulator repeatedly for perturbed values of the design variables [1].

Perturbation is a direct but brute-force method for sensitivity analysis. It is computational expensive and often inaccurate, thus impractical, when the number of circuit parameters for

Manuscript received April 2011; revised August 2011; accepted October 2011. This work was supported by the Research Foundation Flanders (FWO) and by the Italian Ministry of University (MIUR).

Luca De Camillis, Dries Vande Ginste and Daniël De Zutter are with the Department of Information Technology (INTEC), Ghent University, Sint-Pietersnieuwstraat 41, 9000 Ghent Belgium, email: luca.decamillis@UGent.be,vdginste@UGent.be,daniel.dezutter@UGent.be

Francesco Ferranti is with the Department of Information Technology, Internet Based Communication Networks and Services (IBCN), Ghent University - IBBT, Gaston Crommenlaan 8 Bus 201, B-9050 Gent, Belgium, email: francesco.ferranti@ugent.be

Giulio Antonini is with the UAq EMC Laboratory, Dipartimento di Ingeg neria Elettrica e dell'Informazione, Università degli Studi dell'Aquila, Via G Gronchi 18,67100 L'Aquila Italy, e-mail: giulio.antonini@univaq.it. optimization is large. The perturbation approach needs to perturb a design parameter $g$ with $\Delta g$ and therefore to perform a circuit simulation once again in order to get the sensitivities with respect to $g$. Sometimes it may be difficult to select a value for $\Delta g$ to obtain accurate results. If $\Delta g$ is set too large, perturbation itself is inaccurate. If $\Delta g$ is set too small, extremely high simulation accuracy is required to exactly highlight the response difference between $g$ and $g+\Delta g$ [2].

Among EM methods the Partial Element Equivalent Circuit (PEEC) method [3] is becoming increasingly popular since it is able to transform the EM system under examination into a passive RLC equivalent circuit. PEEC uses a circuit interpretation of the Electric Field Integral Equation (EFIE) [4], thus allowing to handle complex problems involving EM fields and circuits [3], [5]-[11]. Nonlinear circuit devices such as drivers and receivers are usually connected with PEEC equivalent circuits using a time-domain circuit simulator (e.g. SPICE [12]).

In this paper, we propose a new method to carry out parameterized sensitivity analysis of systems that depend on multiple design parameters, such as layout and substrate features. The PEEC method is used to compute state-space matrices at a set of design space points. Then, an interpolation process provides parameterized models of these matrices as functions of the design parameters, e.g. geometrical and substrate parameters [13]. The proposed interpolation scheme is able to compute the derivatives of the electromagnetic matrices, which are needed to perform the sensitivity analysis. The proposed algorithm is able to provide sensitivity information over the entire design space of interest, and not only around one operating point.

To be utilized in a time-domain transient simulator, the stability and passivity of the proposed model are fundamental to guarantee stable simulation. Hence, we discuss the required stability and passivity properties of the systems involved in the parameterized sensitivity analysis in this paper.

The paper is organized as follows. Section II describes the PEEC formulation, while Section III discusses the proposed parameterized sensitivity formulation. Section IV presents the interpolation process of the PEEC matrices and Section V discusses the required passivity and stability system properties. Finally, some numerical examples are presented in Section VI to validate the proposed technique. 


\section{PEEC FORMUlation}

In what follows, we describe a quasi-static PEEC formulation [3]. In the standard approach, volumes and surfaces of conductors and dielectrics are discretized into hexahedra and patches respectively, representing elementary regions [11] over which the current and charge densities are expanded into a series of basis functions. Pulse basis functions are usually adopted as expansion and weight functions. Such choice of pulse basis functions corresponds to constant current density and charge density over the elementary volume (inductive) and surface (capacitive) cells, respectively. Following the standard Galerkin's testing procedure, topological elements, namely nodes and branches, are generated and electrical lumped elements are identified modeling both the magnetic and electric field coupling. An example of PEEC electrical quantities for a conductor elementary cell is illustrated, in the Laplace domain, in Fig. 1 where the current controlled voltage sources $s L_{p, i j} I_{j}$ and the current controlled current sources $I_{c c i}$ model the magnetic and electric field coupling, respectively.

The Galerkin's approach is applied to convert the continuous electromagnetic problem described by the EFIE to a discrete problem in terms of electrical circuit quantities, i.e. currents $\boldsymbol{i}(t)$ and node potentials $\boldsymbol{v}(t)$. Let us denote with $n_{n}$ the number of the nodes and with $n_{i}$ the number of branches where currents flow. Among this latter, $n_{c}$ and $n_{d}$ represent the branches of conductors and dielectrics, respectively. Furthermore, let us assume to be interested in generating an admittance representation having $n_{p}$ output currents $\boldsymbol{i}_{p}(t)$ under voltage excitation $\boldsymbol{v}_{p}(t)$. Using the Modified Nodal Approach (MNA) [14], the following admittance formulation is obtained [13], [15]:

$$
\begin{aligned}
& \underbrace{\left[\begin{array}{cccc}
\mathbf{P} & \mathbf{0}_{n_{n}, n_{i}} & \mathbf{0}_{n_{n}, n_{d}} & \mathbf{0}_{n_{n}, n_{p}} \\
\mathbf{0}_{n_{i}, n_{n}} & \mathbf{L}_{p} & \mathbf{0}_{n_{i}, n_{d}} & \mathbf{0}_{n_{i}, n_{p}} \\
\mathbf{0}_{n_{d}, n_{n}} & \mathbf{0}_{n_{d}, n_{i}} & \mathbf{C}_{d} & \mathbf{0}_{n_{d}, n_{p}} \\
\mathbf{0}_{n_{p}, n_{n}} & \mathbf{0}_{n_{p}, n_{i}} & \mathbf{0}_{n_{p}, n_{d}} & \mathbf{0}_{n_{p}, n_{p}}
\end{array}\right]}_{\mathbf{C}} \frac{d}{d t} \underbrace{\left[\begin{array}{c}
\boldsymbol{q}(t) \\
\boldsymbol{i}(t) \\
\boldsymbol{v}_{d}(t) \\
\boldsymbol{i}_{k}(t)
\end{array}\right]}_{\boldsymbol{x}(t)}= \\
& -\underbrace{\left[\begin{array}{cccc}
\mathbf{0}_{n_{n}, n_{n}} & -\mathbf{P A}^{\mathrm{T}} & \mathbf{0}_{n_{n}, n_{d}} & \mathbf{P K}^{\mathrm{T}} \\
\mathbf{A P} & \mathbf{R} & \boldsymbol{\Phi} & \mathbf{0}_{n_{i}, n_{p}} \\
\mathbf{0}_{n_{d}, n_{n}} & -\boldsymbol{\Phi}^{\mathrm{T}} & \mathbf{0}_{n_{d}, n_{d}} & \mathbf{0}_{n_{d}, n_{p}} \\
-\mathbf{K P} & \mathbf{0}_{n_{p}, n_{i}} & \mathbf{0}_{n_{p}, n_{d}} & \mathbf{0}_{n_{p}, n_{p}}
\end{array}\right]}_{\mathbf{G}} \cdot \underbrace{\left[\begin{array}{c}
\boldsymbol{q}(t) \\
\boldsymbol{i}(t) \\
\boldsymbol{v}_{d}(t) \\
\boldsymbol{i}_{k}(t)
\end{array}\right]}_{\boldsymbol{x}(t)}+ \\
& \underbrace{\left[\begin{array}{c}
\mathbf{0}_{n_{n}+n_{i}+n_{d}, n_{p}} \\
-\mathbf{I}_{n_{p}, n_{p}}
\end{array}\right]}_{\mathbf{B}} \cdot \underbrace{\left[\boldsymbol{v}_{p}(t)\right]}_{\boldsymbol{u}(t)}
\end{aligned}
$$

where $\mathbf{P} \in \mathbb{R}^{n_{n} \times n_{n}}$ and $\mathbf{L}_{p} \in \mathbb{R}^{n_{i} \times n_{i}}$ are the coefficients of potential and partial inductance matrices, respectively, $\mathbf{R} \in$ $\mathbb{R}^{n_{i} \times n_{i}}$ is a diagonal matrix containing the resistances of volume cells and $\mathbf{C}_{d} \in \mathbb{R}^{n_{d} \times n_{d}}$ is the excess capacitance matrix describing the polarization charge in dielectrics [16]. $\mathbf{A} \in \mathbb{R}^{n_{i} \times n_{n}}$ is the connectivity matrix, while $\mathbf{K} \in \mathbb{R}^{n_{p} \times n_{n}}$ is a selection matrix introduced to define the port voltages in terms of node potentials:

$$
\boldsymbol{v}_{p}(t)=\mathbf{K} \boldsymbol{v}(t)
$$

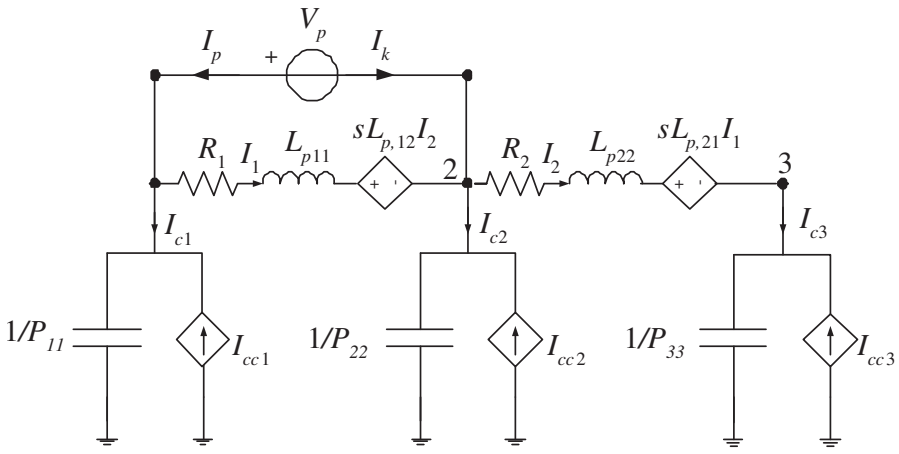

Fig. 1. Illustration of PEEC circuit electrical quantities for a conductor elementary cell.

TABLE I

SCALED UNITS

\begin{tabular}{|c|c|}
\hline Voltage & $\mathrm{V}$ \\
Current & $\mathrm{mA}$ \\
Charge & $\mathrm{pC}$ \\
$P$ & $\mathrm{pF}^{-1}$ \\
$C_{d}$ & $\mathrm{pF}$ \\
$R$ & $\mathrm{k} \Omega$ \\
$L_{p}$ & $\mu \mathrm{H}$ \\
$f$ & $\mathrm{GHz}$ \\
$s$ & $\mathrm{~ns}$ \\
\hline
\end{tabular}

In (1), $\boldsymbol{q}(t) \in \mathbb{R}^{n_{n} \times 1}$ represents the charges on the conductors, $\boldsymbol{i}(t) \in \mathbb{R}^{n_{i} \times 1}$ is the vector of volume currents, $\boldsymbol{v}_{d}(t) \in \mathbb{R}^{n_{d} \times 1}$ describes the voltage drop across the excess capacitance and $\boldsymbol{i}_{k}(t) \in \mathbb{R}^{n_{p} \times 1}$ represents the port currents. $\mathbf{I}_{n_{p}, n_{p}}$ is the identity matrix of dimension equal to the number of ports and $\Phi$ is

$$
\boldsymbol{\Phi}=\left[\begin{array}{c}
\mathbf{0}_{n_{c}, n_{d}} \\
\mathbf{I}_{n_{d}, n_{d}}
\end{array}\right]
$$

In a more compact form, according to [17], the previous equations (1) can be rewritten as

$$
\begin{aligned}
\mathbf{C} \dot{\boldsymbol{x}}(t) & =-\mathbf{G} \boldsymbol{x}(t)+\mathbf{B} \boldsymbol{u}(t) \\
\boldsymbol{i}_{p}(t) & =\mathbf{L}^{\mathrm{T}} \boldsymbol{x}(t)
\end{aligned}
$$

where $\mathbf{C} \in \mathbb{R}^{n_{u} \times n_{u}}, \mathbf{G} \in \mathbb{R}^{n_{u} \times n_{u}}, \mathbf{B} \in \mathbb{R}^{n_{u} \times n_{p}}, \mathbf{L}=\mathbf{B}$, $\boldsymbol{x}(t)=\left[\boldsymbol{q}(t) \boldsymbol{i}(t) \boldsymbol{v}_{d}(t) \boldsymbol{i}_{k}(t)\right]^{\mathrm{T}} \in \mathbb{R}^{n_{u} \times 1}$ and $n_{u}=n_{n}+n_{i}+$ $n_{d}+n_{p}$ is the number of state variables [13], [15]. The vector $\boldsymbol{i}_{p}(t)$ describes the $n_{p}$ port currents that are of opposite sign with respect to $\boldsymbol{i}_{k}(t)$ [17]. So, this is an $n_{p}$-port formulation, whereby the only sources are the voltage sources at the $n_{p^{-}}$ port's nodes.

\section{A. Scaling}

The system of equations (1) is typically ill-conditioned because the values of charges are usually much smaller than those of currents and voltages. Correspondingly, the entries of the matrix $\mathbf{P}$ are larger than the other elements in matrices $\mathbf{C}$ and $\mathbf{G}$ by several orders of magnitude. In order to mitigate such a problem, scaling can be adopted [13]. The units of the electrical quantities are changed consistently as shown in Table I. 


\section{SENSITIVITY FORMULATION}

The proposed technique aims at performing parameterized sensitivity analyses of systems that depend on multiple design parameters, such as layout and substrate features. The PEEC method provides an admittance formulation in a rational form, which leads to a time-domain model that can be efficiently interfaced with linear and nonlinear terminations or SPICElike simulators. In what follows, we describe the proposed algorithm and define the sensitivity formulation.

The system of equations (4) is in a descriptor form with a singular descriptor matrix $\mathbf{C}$. Hence, $\mathbf{C}$ cannot be inverted. Therefore, to solve the differential equation (4), we decompose the overall system into two subsystem whose unknowns are:

$$
\boldsymbol{x}_{1}=\left[\begin{array}{c}
\boldsymbol{q}(t, \boldsymbol{g}) \\
\boldsymbol{i}(t, \boldsymbol{g}) \\
\boldsymbol{v}_{d}(t, \boldsymbol{g})
\end{array}\right] \quad \boldsymbol{x}_{2}=\left[\boldsymbol{i}_{k}(t, \boldsymbol{g})\right]
$$

Thus (4) is recast as

$$
\begin{gathered}
{\left[\begin{array}{cc}
\mathbf{C}_{11}(\boldsymbol{g}) & \mathbf{C}_{12}(\boldsymbol{g}) \\
\mathbf{C}_{21}(\boldsymbol{g}) & \mathbf{C}_{22}(\boldsymbol{g})
\end{array}\right]\left[\begin{array}{l}
\dot{\boldsymbol{x}}_{1}(t, \boldsymbol{g}) \\
\dot{\boldsymbol{x}}_{2}(t, \boldsymbol{g})
\end{array}\right]=} \\
-\left[\begin{array}{ll}
\mathbf{G}_{11}(\boldsymbol{g}) & \mathbf{G}_{12}(\boldsymbol{g}) \\
\mathbf{G}_{21}(\boldsymbol{g}) & \mathbf{G}_{22}(\boldsymbol{g})
\end{array}\right]\left[\begin{array}{l}
\boldsymbol{x}_{1}(t, \boldsymbol{g}) \\
\boldsymbol{x}_{2}(t, \boldsymbol{g})
\end{array}\right]+\left[\begin{array}{l}
\mathbf{B}_{1}(\boldsymbol{g}) \\
\mathbf{B}_{2}(\boldsymbol{g})
\end{array}\right] \boldsymbol{v}_{p}(t, \boldsymbol{g})
\end{gathered}
$$

$$
\boldsymbol{i}_{p}(t, \boldsymbol{g})=\left[\begin{array}{c}
\mathbf{L}_{1}(\boldsymbol{g}) \\
\mathbf{L}_{2}(\boldsymbol{g})
\end{array}\right]^{T}\left[\begin{array}{l}
\boldsymbol{x}_{1}(t, \boldsymbol{g}) \\
\boldsymbol{x}_{2}(t, \boldsymbol{g})
\end{array}\right]
$$

where $\mathbf{C}_{11}$ and $\mathbf{G}_{11} \in \mathbb{R}^{n_{s} \times n_{s}}, \mathbf{C}_{12}, \mathbf{G}_{12}, \mathbf{B}_{1}$ and $\mathbf{L}_{1} \in$ $\mathbb{R}^{n_{s} \times n_{p}}, \mathbf{C}_{21}$ and $\mathbf{G}_{21} \in \mathbb{R}^{n_{p} \times n_{s}}, \mathbf{C}_{22}, \mathbf{G}_{22}, \mathbf{B}_{2}$ and $\mathbf{L}_{2} \in$ $\mathbb{R}^{n_{p} \times n_{p}}$, where $n_{s}=n_{u}-n_{p}$. We have now also explicitly introduced the dependence from the $M$ design parameters $\boldsymbol{g}=$ $\left(g^{(m)}\right)_{m=1}^{M}$. Substituting the actual values of (4) into (6), we obtain:

$$
\begin{aligned}
& {\left[\begin{array}{cc}
\mathbf{C}_{11}(\boldsymbol{g}) & \mathbf{0} \\
\mathbf{0} & \mathbf{0}
\end{array}\right]\left[\begin{array}{c}
\dot{\boldsymbol{x}}_{1}(t, \boldsymbol{g}) \\
\dot{\boldsymbol{i}}_{k}(t, \boldsymbol{g})
\end{array}\right]=} \\
& -\left[\begin{array}{cc}
\mathbf{G}_{11}(\boldsymbol{g}) & \mathbf{G}_{12}(\boldsymbol{g}) \\
\mathbf{G}_{21}(\boldsymbol{g}) & \mathbf{0}
\end{array}\right]\left[\begin{array}{c}
\boldsymbol{x}_{1}(t, \boldsymbol{g}) \\
\boldsymbol{i}_{k}(t, \boldsymbol{g})
\end{array}\right]+\left[\begin{array}{c}
\mathbf{0} \\
-\mathbf{I}
\end{array}\right] \boldsymbol{v}_{p}(t, \boldsymbol{g})
\end{aligned}
$$

$$
\boldsymbol{i}_{p}(t, \boldsymbol{g})=\left[\begin{array}{ll}
\mathbf{0} & -\mathbf{I}
\end{array}\right]\left[\begin{array}{c}
\boldsymbol{x}_{1}(t, \boldsymbol{g}) \\
\boldsymbol{i}_{k}(t, \boldsymbol{g})
\end{array}\right]
$$

Equations (7a) and (7b) can be split into three sets of equations,

$$
\left\{\begin{aligned}
\mathbf{C}_{11}(\boldsymbol{g}) \dot{\boldsymbol{x}}_{1}(t, \boldsymbol{g}) & =-\mathbf{G}_{11}(\boldsymbol{g}) \boldsymbol{x}_{1}(t, \boldsymbol{g})-\mathbf{G}_{12}(\boldsymbol{g}) \boldsymbol{i}_{k}(t, \boldsymbol{g}) \\
\mathbf{0} & =-\mathbf{G}_{21}(\boldsymbol{g}) \boldsymbol{x}_{1}(t, \boldsymbol{g})-\boldsymbol{v}_{p}(t, \boldsymbol{g}) \\
\boldsymbol{i}_{p}(t, \boldsymbol{g}) & =-\boldsymbol{i}_{k}(t, \boldsymbol{g})
\end{aligned}\right.
$$

which can be rewritten in a more compact form as:

$$
\left\{\begin{aligned}
\mathbf{C}_{11}(\boldsymbol{g}) \dot{\boldsymbol{x}}_{1}(t, \boldsymbol{g}) & =-\mathbf{G}_{11}(\boldsymbol{g}) \boldsymbol{x}_{1}(t, \boldsymbol{g})+\mathbf{G}_{12}(\boldsymbol{g}) \boldsymbol{i}_{p}(t, \boldsymbol{g}) \\
\boldsymbol{v}_{p}(t, \boldsymbol{g}) & =\mathbf{G}_{12}^{\mathrm{T}}(\boldsymbol{g}) \boldsymbol{x}_{1}(t, \boldsymbol{g})
\end{aligned}\right.
$$

where we have considered $\mathbf{G}_{12}=-\left(\mathbf{G}_{21}\right)^{\mathrm{T}}$ by inspection of (1) and (6) and taking into account $\mathbf{P}=\mathbf{P}^{\mathrm{T}}$ [13]. Equation (9) needs to be terminated with appropriate termination conditions in order to compute the state-space system itself. It is worth to notice that the first formulation (4) represents the system in an admittance form, while the second one (9) adopts an impedance form. In fact, in the Laplace domain using the complex argument $s$ and capital letters for the voltages and current to distinguish from time domain, (9) is recast as:

$$
\mathbf{V}_{p}(s, \boldsymbol{g})=\mathbf{Z}(s, \boldsymbol{g}) \mathbf{I}_{p}(s, \boldsymbol{g})
$$

where:

$$
\mathbf{Z}(s, \boldsymbol{g})=\mathbf{G}_{12}^{\mathrm{T}}(\boldsymbol{g})\left(s \mathbf{C}_{11}(\boldsymbol{g})+\mathbf{G}_{11}(\boldsymbol{g})\right)^{-1} \mathbf{G}_{12}(\boldsymbol{g})
$$

In the next section we will focus on the PEEC matrix derivatives; those matrices, as will be shown, are needed in the calculus of the sensitivity. Thereafter, a sensitivity formulation, with RC-termination conditions, will be derived from (9). For ease of notation, we discuss the parameterized sensitivity analysis with respect to one design parameter $\boldsymbol{g}=g^{1}=g$ $(M=1)$. Generalization of the formalism to the multivariate case $(M \neq 1)$ is straightforward.

\section{A. Matrix derivatives}

We assume that a topologically fixed discretization mesh is used and that it is independent from the specific design parameter values. To guarantee the accuracy of the method, we choose the finest meshing over all the geometrical parameter configurations as follows. First, all the possible meshes are computed accordingly to the $\lambda_{\min } / 20$ rule, where $\lambda_{\min }$ is the wavelength corresponding to the maximum frequency of interest. Next, the mesh with the highest number of cells is selected. This leads to an overmeshing for some of the configurations (which would have required a lower meshing), but this is necessary to preserve the number of unknowns, i.e. the PEEC matrices dimensions, while maintaining accuracy. Thus, keeping the same initial mesh for all the layout configurations, when shape parameters are modified, the mesh is only locally stretched or shrunk. In general, the global coordinates of the nodes, as well as the length and orientation of the edges of the topologically fixed mesh change when shape parameters change; however, these changes are neither introducing new state variables nor eliminating existing state variables. The matrices $\mathbf{B}$ and $\mathbf{L}$ are uniquely determined by the circuit topology and therefore remain unchanged, while the matrices $\mathbf{C}$ and $\mathbf{G}$ are defined as functions of the design parameters. At a deeper level in the MNA equations (1), the previous assumptions lead to $\mathbf{P}(g), \mathbf{L}_{p}(g), \mathbf{C}_{d}(g), \mathbf{R}(g)$, while the other internal PEEC matrices $\mathbf{A}, \boldsymbol{\Phi}, \mathbf{K}$ are not dependent on $g$.

Therefore, the derivatives of the matrices in (9) can be expressed as:

$$
\begin{gathered}
\widehat{\mathbf{C}}_{11}(g)=\left[\begin{array}{ccc}
\widehat{\mathbf{P}}(g) & \mathbf{0}_{n_{n}, n_{i}} & \mathbf{0}_{n_{n}, n_{d}} \\
\mathbf{0}_{n_{i}, n_{n}} & \widehat{\mathbf{L}}_{p}(g) & \mathbf{0}_{n_{i}, n_{d}} \\
\mathbf{0}_{n_{d}, n_{n}} & \mathbf{0}_{n_{d}, n_{i}} & \widehat{\mathbf{C}}_{d}(g)
\end{array}\right] \\
\widehat{\mathbf{G}}_{11}(g)=\left[\begin{array}{ccc}
\mathbf{0}_{n_{n}, n_{n}} & -\widehat{\mathbf{P}}(g) \mathbf{A}^{\mathrm{T}} & \mathbf{0}_{n_{n}, n_{d}} \\
\mathbf{A} \widehat{\mathbf{P}}(g) & \widehat{\mathbf{R}}(g) & \mathbf{0}_{n_{i}, n_{d}} \\
\mathbf{0}_{n_{d}, n_{n}} & \mathbf{0}_{n_{i}, n_{d}} & \mathbf{0}_{n_{d}, n_{d}}
\end{array}\right] \\
\widehat{\mathbf{G}}_{12}(g)=\left[\begin{array}{c}
\widehat{\mathbf{P}}(g) \mathbf{K}^{\mathrm{T}} \\
\mathbf{0}_{n_{i}, n_{p}} \\
\mathbf{0}_{n_{d}, n_{p}}
\end{array}\right]
\end{gathered}
$$




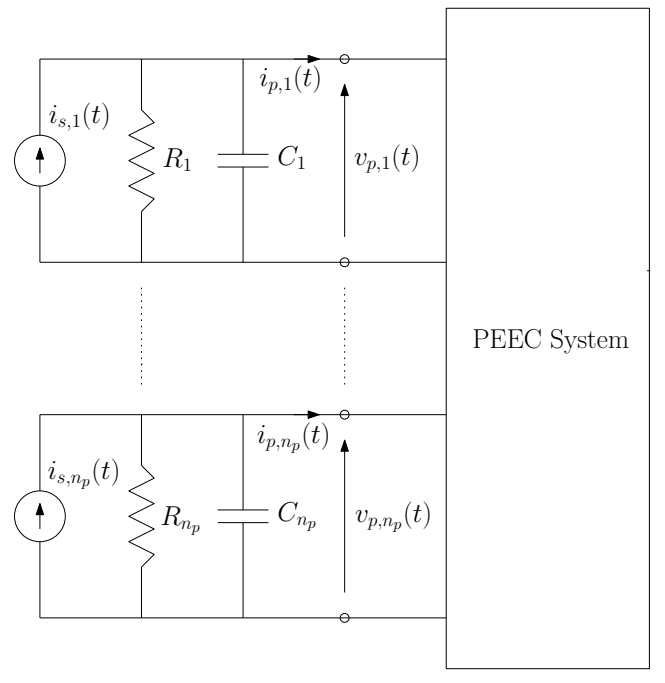

Fig. 2. RC terminations of the PEEC model

where the hat notation $\widehat{f}$ denotes the derivative of the function $f$ with respect to the parameter $g$.

\section{B. RC terminations}

In what follows, the computation of current and voltage sensitivity will be discussed in the case of RC terminations. The port currents can be expressed as:

$$
\boldsymbol{i}_{p}(t, g)=\boldsymbol{i}_{s}(t)-\mathbf{G}_{T} \boldsymbol{v}_{p}(t, g)-\mathbf{C}_{T} \frac{\mathrm{d} \boldsymbol{v}_{p}(t, g)}{\mathrm{d} t}
$$

where $\boldsymbol{i}_{s}(t) \in \mathbb{R}^{n_{p} \times 1}$ is the current source vector and $\mathbf{G}_{T}$ and $\mathbf{C}_{T} \in \mathbb{R}^{n_{p} \times n_{p}}$ are diagonal matrices, whose diagonal elements describe linear resistive and capacitive lumped elements. The equivalent circuit of (13) is provided in Fig. 2. Inserting (13) into (9), yields:

$$
\begin{aligned}
& {\left[\mathbf{C}_{11}(g)+\mathbf{G}_{12}(g) \mathbf{C}_{T} \mathbf{G}_{12}^{\mathrm{T}}(g)\right] \dot{\boldsymbol{x}}_{1}(t, g)=} \\
- & {\left[\mathbf{G}_{11}(g)+\mathbf{G}_{12}(g) \mathbf{G}_{T} \mathbf{G}_{12}^{\mathrm{T}}(g)\right] \boldsymbol{x}_{1}(t, g)+\mathbf{G}_{12}(g) \boldsymbol{i}_{s}(t) }
\end{aligned}
$$

$$
\begin{aligned}
\boldsymbol{v}_{p}(t, g) & =\mathbf{G}_{12}^{\mathrm{T}}(g) \boldsymbol{x}_{1}(t, g) \\
\boldsymbol{i}_{p}(t, g) & =\boldsymbol{i}_{s}(t)-\mathbf{G}_{T} \mathbf{G}_{12}^{\mathrm{T}}(g) \boldsymbol{x}_{1}(t, g)-\mathbf{C}_{T} \mathbf{G}_{12}^{\mathrm{T}}(g) \dot{\boldsymbol{x}}_{1}(t, g)
\end{aligned}
$$

The current and voltage sensitivities are obtained by differentiating (14) with respect to the parameter $g$ :

$$
\begin{gathered}
{\left[\mathbf{C}_{11}(g)+\mathbf{G}_{12}(g) \mathbf{C}_{T} \mathbf{G}_{12}^{\mathrm{T}}(g)\right] \widehat{\dot{\boldsymbol{x}}}_{1}(t, g)=} \\
-\left[\mathbf{G}_{11}(g)+\mathbf{G}_{12}(g) \mathbf{G}_{T} \mathbf{G}_{12}^{\mathrm{T}}(g)\right] \widehat{\boldsymbol{x}}_{1}(t, g) \\
-\left[\widehat{\mathbf{C}}_{11}(g)+\widehat{\mathbf{G}}_{12}(g) \mathbf{C}_{T} \mathbf{G}_{12}^{\mathrm{T}}(g)+\mathbf{G}_{12} \mathbf{C}_{T} \widehat{\mathbf{G}}_{12}^{\mathrm{T}}\right] \dot{\boldsymbol{x}}_{1}(t, g) \\
-\left[\widehat{\mathbf{G}}_{11}(g)+\widehat{\mathbf{G}}_{12}(g) \mathbf{G}_{T} \mathbf{G}_{12}^{\mathrm{T}}(g)+\mathbf{G}_{12}(g) \mathbf{G}_{T} \widehat{\mathbf{G}}_{12}^{\mathrm{T}}(g)\right] \boldsymbol{x}_{1}(t, g) \\
+ \\
+\widehat{\mathbf{G}}_{12}(g) \boldsymbol{i}_{s}(t)
\end{gathered}
$$

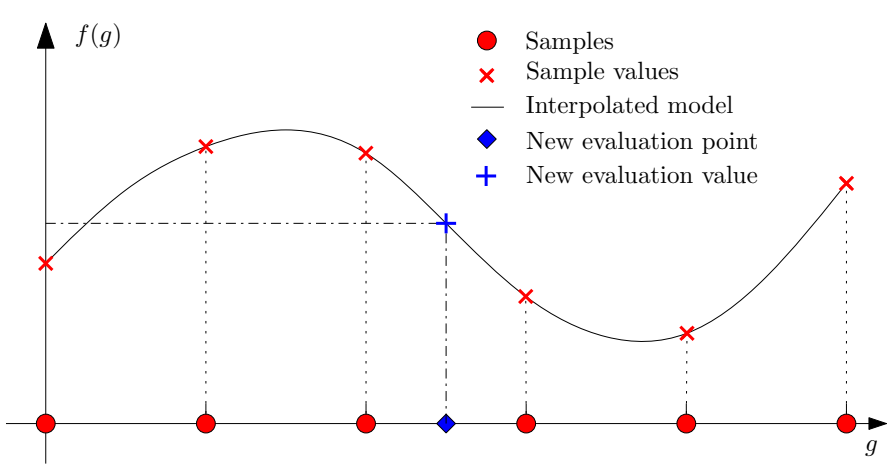

Fig. 3. Example of sampling design space grid.

$$
\begin{aligned}
\widehat{\boldsymbol{v}}_{p}(t, g)= & \widehat{\mathbf{G}}_{12}^{\mathrm{T}}(g) \boldsymbol{x}_{1}(t, g)+\mathbf{G}_{12}^{\mathrm{T}}(g) \widehat{\boldsymbol{x}}_{1}(t, g) \\
\widehat{\boldsymbol{i}}_{p}(t, g)= & -\mathbf{G}_{T}\left[\widehat{\mathbf{G}}_{12}^{\mathrm{T}}(g) \boldsymbol{x}_{1}(t, g)+\mathbf{G}_{12}^{\mathrm{T}}(g) \widehat{\boldsymbol{x}}_{1}(t, g)\right] \\
& -\mathbf{C}_{T}\left[\widehat{\mathbf{G}}_{12}^{\mathrm{T}}(g) \dot{\boldsymbol{x}}_{1}(t, g)+\mathbf{G}_{12}^{\mathrm{T}}(g) \widehat{\boldsymbol{x}}_{1}(t, g)\right]
\end{aligned}
$$

The contributions $\boldsymbol{x}_{1}(t, g)$ and $\dot{\boldsymbol{x}}_{1}(t, g)$ in (15) are computed from (14). Upon knowledge of the matrices (12), computed as explained below (Section IV), (15) can be calculated by means of any differential equation solver.

\section{INTERPOLATION}

A set of multivariate data samples $\left\{\boldsymbol{g}_{k}, \mathbf{P}\left(\boldsymbol{g}_{k}\right)\right.$, $\left.\mathbf{L}_{p}\left(\boldsymbol{g}_{k}\right), \mathbf{C}_{d}\left(\boldsymbol{g}_{k}\right), \mathbf{R}\left(\boldsymbol{g}_{k}\right), \mathbf{K}_{r}\left(\boldsymbol{g}_{k}\right)\right\}_{k=1}^{K_{t o t}}$ is gathered to build an interpolation model. An example is provided in Fig. 3 for the case of one parameter. The red dots ( $\circ$ ) represent the sampling points used to build the interpolation model; for each red dot a set of PEEC matrices is computed. In the proposed approach a uniformly spaced sampling grid is utilized. In the N-dimensional (ND) case, the points are still uniformly sampled, but in the different directions of the ND design space. Next, a model covering the entire design space, is built by means of interpolation schemes (full black line). We use the multivariate cubic spline interpolation method [18], which is well-known for its stable and smooth characteristics. The proposed interpolation scheme is continuous in the first and second order derivatives. Now, a new set of PEEC matrices and their derivatives can be obtained by evaluating the interpolation model in any point of the design space. In Fig. 3, the blue diamond $(\diamond)$ represents such a new sample.

\section{STABILITY AND PASSIVITY PROPERTIES}

Stability and passivity are crucial when the interpolation model is utilized in a circuit simulator for transient analysis. It is known that, while a passive system is also stable, the reverse is not necessarily true. Passivity refers to the property of systems that cannot generate more energy than they absorb through their electrical ports. When the system is terminated by arbitrary passive loads, none of them will cause the system to become unstable. In the Laplace domain, a linear network described by an admittance matrix $\mathbf{Z}(s)$ is passive if [19]:

1) $\mathbf{Z}\left(s^{*}\right)=\mathbf{Z}^{*}(s)$ for all $s$, where "*" is the complex conjugate operator. 
2) $\mathbf{Z}(s)$ is analytic in $\Re e(s)>0$.

3) $\mathbf{Z}(s)$ is a positive-real matrix, i.e. :

$\mathbf{z}^{* T}\left(\mathbf{Z}^{T}\left(s^{*}\right)+\mathbf{Z}(s)\right) \mathbf{z} \geq 0 ; \forall s: \Re e(s)>0$ and any arbitrary vector $\mathbf{z}$.

For each sample of the sampling grid, the computed internal PEEC matrices $\mathbf{P}(\boldsymbol{g}), \mathbf{L}_{p}(\boldsymbol{g})$ and $\mathbf{C}_{d}(\boldsymbol{g})$ are positive definite and $\mathbf{R}(\boldsymbol{g})$ is positive semidefinite [13], [15]. Hence, the impedance system described in (10), with $\mathbf{Z}=$ $\mathbf{G}_{12}^{\mathrm{T}}\left({ }_{s} \mathbf{C}_{11}+\mathbf{G}_{11}\right)^{-1} \mathbf{G}_{12}$ (11) can easily be proven to be a passive system, following the procedure outlined in [20]. The multivariate interpolation models of the internal PEEC matrices must now also preserve the positive definiteness or positive semidefiniteness to guarantee passivity of (9) for each design space point outside the sampling grid. To this aim, we use the spline-based passivity-preserving interpolation methods described in [13], which use particular mapping functions to guarantee overall passivity, and also allow to analytically compute the derivative matrices in (12). This passivity in the Laplace domain leads to stable time-domain simulations for the systems (9) for arbitrary passive loads.

To prove the stability of the sensitivity system for arbitrary passive loads, we can derive the expression of the sensitivity by deriving (10) with respect to a parameter of interest $g$ :

$$
\widehat{\mathbf{V}}_{p}=\mathbf{Z} \widehat{\mathbf{I}}_{p}+\widehat{\mathbf{Z}} \mathbf{I}_{p}
$$

To prove the stability of (16), the two terms of this equation have to be considered. The stability of the first term has just been discussed, in what follows we will discuss the stability of the second term $\widehat{\mathbf{Z}} \mathbf{I}_{p}$. As we can see, the port currents $\mathbf{I}_{p}$ are obtained by the solution of (10), together with the corresponding termination equations, which yields a stable result. Thus, the stability of $\widehat{\mathbf{Z}}$ is sufficient to guarantee stable time-domain simulations. The derivative of the impedance system can written as:

$$
\begin{aligned}
& \widehat{\mathbf{Z}}=\widehat{\mathbf{G}}_{12}^{\mathrm{T}}\left(s \mathbf{C}_{11}+\mathbf{G}_{11}\right)^{-1} \mathbf{G}_{12}+ \\
& \mathbf{G}_{12}^{\mathrm{T}} \frac{\mathrm{d}}{\mathrm{d} g}\left(s \mathbf{C}_{11}+\mathbf{G}_{11}\right)^{-1} \mathbf{G}_{12} \\
& \quad+\mathbf{G}_{12}^{\mathrm{T}}\left(s \mathbf{C}_{11}+\mathbf{G}_{11}\right)^{-1} \widehat{\mathbf{G}}_{12}
\end{aligned}
$$

Since the derivative of an inverse matrix can be simplified as:

$$
\frac{\mathrm{d} \mathbf{A}^{-1}}{\mathrm{~d} x}=-\mathbf{A}^{-1} \frac{\mathrm{d} \mathbf{A}}{\mathrm{d} x} \mathbf{A}^{-1}
$$

equation (17) can be recast as:

$$
\begin{aligned}
& \widehat{\mathbf{Z}}=\widehat{\mathbf{G}}_{12}^{\mathrm{T}}\left(s \mathbf{C}_{11}+\mathbf{G}_{11}\right)^{-1} \mathbf{G}_{12}- \\
& \mathbf{G}_{12}^{\mathrm{T}}\left(s \mathbf{C}_{11}+\mathbf{G}_{11}\right)^{-1}\left(s \widehat{\mathbf{C}}_{11}+\widehat{\mathbf{G}}_{11}\right)\left(s \mathbf{C}_{11}+\mathbf{G}_{11}\right)^{-1} \mathbf{G}_{12} \\
& \quad+\mathbf{G}_{12}^{\mathrm{T}}\left(s \mathbf{C}_{11}+\mathbf{G}_{11}\right)^{-1} \widehat{\mathbf{G}}_{12}
\end{aligned}
$$

We notice that the poles of (19) depend on the characteristic polynomial of $\left(s \mathbf{C}_{11}+\mathbf{G}_{11}\right)$ and therefore they are the same poles of (11) with double multiplicity. Since (11) represents a passive and stable system, we can obviously conclude that (19) is a stable system. We finally state that the sensitivity system (16), as a sum of two stable contributions, has a stable response for arbitrary passive loads.

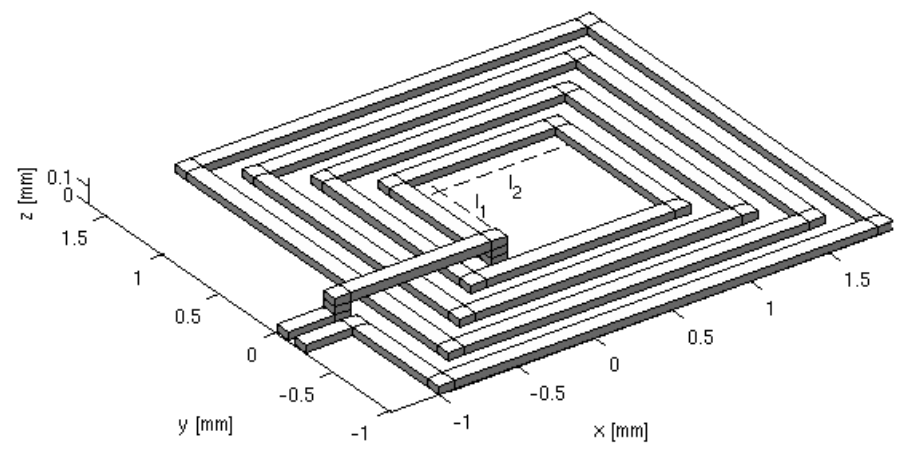

Fig. 4. Spiral inductor.

\section{NumERICAL RESUltS}

Two numerical examples are proposed to validate the discussed technique. Parameterized time-domain sensitivity analysis is performed in both cases. Time-domain results obtained by the presented algorithm are compared with those obtained using the perturbative approach (with respect to the parameter $\left.g_{m}\right)$ :

$$
\widehat{\boldsymbol{v}}_{p, g_{m}}(t, \boldsymbol{g})=\frac{\boldsymbol{v}_{p}\left(t, g_{1}, . ., g_{m}+\Delta g_{m}, . ., g_{M}\right)-\boldsymbol{v}_{p}(t, \boldsymbol{g})}{\Delta g_{m}}
$$

where $m=1, \ldots, M$ and $\Delta g_{m}$ represents the perturbation. The accuracy of the perturbative approach depends on the choice of the increment $\Delta g_{m}$ : if the increment is not small enough, the estimation of the derivative is not accurate, while if the perturbation is very small compared with the nominal value, numerical problems may occur due to numerical noise. This may lead to inaccurate computation of the system sensitivities. In contrast, thanks to the interpolation model, the method presented in this paper leads to more accuracy and numerical stability since the derivatives are computed from continuously differentiable polynomials, built by means of spline functions. The numerical simulations have been performed on a Linux platform on an Intel Core(TM) i5 CPU 2.67 GHz machines with 8 GB RAM.

\section{A. Spiral inductor}

A spiral inductor, shown in Fig. 4, has been modeled in this example. The dimensions are $l_{1}=0.8 \mathrm{~mm}$ (length of first edge), $l_{2}=1.12 \mathrm{~mm}$ (length of second edge), $t=0.05 \mathrm{~mm}$ (thickness of the spiral), $n=32$ (number of edges). A trivariate model has been built as a function of the conductor width $w \in[0.10-0.15] \mathrm{mm}$ and the space between turns $s \in[0.15-0.30] \mathrm{mm}$ in addition to time. A uniform sampling grid has been built for six values of $w$ and six values of $s$, resulting in a 36 sets of PEEC matrices. Then, the interpolation process, discussed in Section IV, has been performed.

The input port has been excited by a smooth pulse voltage source with amplitude $1 \mathrm{~V}$, rise/fall times $\tau_{r}=\tau_{f}=1.5 \mathrm{~ns}$, width $7 \mathrm{~ns}$ and internal resistance $R_{T}=50 \Omega$. Parameterized voltage sensitivities with respect to the two parameters have been computed with the proposed and perturbative approaches for five values of $w$, equidistantly spaced between $0.105 \mathrm{~mm}$ 


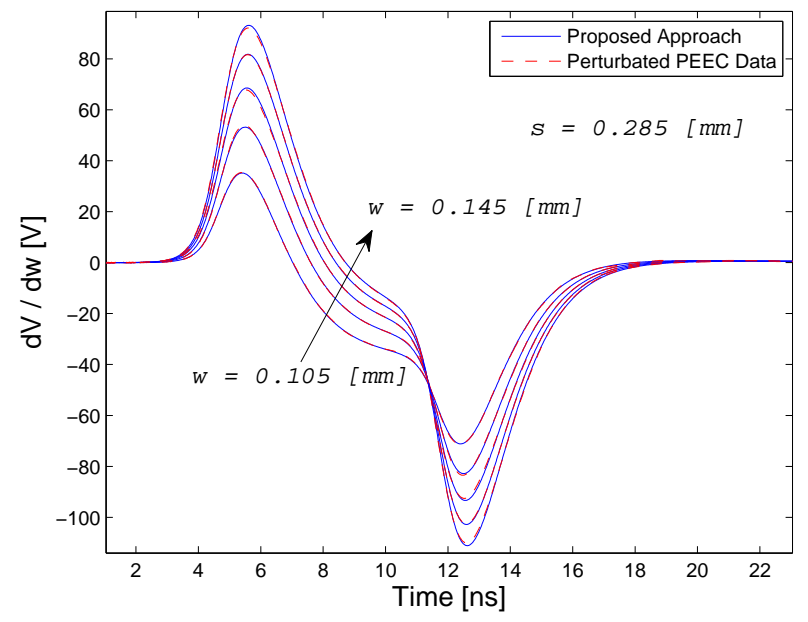

Fig. 5. Time-domain voltage sensitivity with respect to $w$ for the spiral inductor (Section VI-A)

and $0.145 \mathrm{~mm}$, and for five values of $s$, equidistantly spaced between $0.165 \mathrm{~mm}$ and $0.285 \mathrm{~mm}$. So, these values were chosen outside the sampling grid in order to have a fair accuracy comparison. Figs. 5 and 6 show the sensitivities for some of those values. As clearly seen, the agreement between the proposed and perturbative approach is satisfactory.

Table II compares the computational costs between the perturbative and proposed method. Here, the sensitivity in one design space point with respect to the two parameters has been calculated. In this particular case, to obtain the parameterized model 36 PEEC simulations were used (51 s per simulation) and it took $21 \mathrm{~s}$ to interpolate the data. Using this model, only $56.6 \mathrm{~s}$ are needed to perform the time-domain sensitivity simulation in one design space point with respect to the two parameters, therefore for $N$ points $(36 \cdot 51+21+56.6 \cdot N) \mathrm{s}$ are needed. In the perturbative approach, 3 PEEC simulations per point are needed (one nominal value and one neighbor per parameter) and $9.3 \mathrm{~s}$ to perform the time-domain sensitivity simulation, leading to $(3 \cdot 51+9.3) \cdot N \mathrm{~s}$ for $N$ points. From this two-dimensional example, it is clear that our new approach become advantageous for $N \geq 18$. It can be seen that the proposed method is nearly three times faster then the perturbative approach, apart from the computational overhead required for the generation of the interpolation models. This cost can be neglected for a large number of sensitivity computations, for example if the proposed method is inserted in an optimization process and it has to be invoked many times. Using the proposed technique, different optimization processes can be easily performed on a specific system, e.g. when different sets of terminations (drivers and receivers) are connected to the ports or when the source specifications change.

\section{B. Three-port microstrip power-divider circuit}

A three-port microstrip power-divider circuit [21] has been modeled in this example. It is shown in Fig. 7. The geometrical dimensions are $l_{X}=20 \mathrm{~mm}, l_{Y}=20 \mathrm{~mm}, l_{Y, 1}=7.2 \mathrm{~mm}$, $l_{Y, 3}=15.2 \mathrm{~mm}$, the conductor thickness $t_{C o n d}=0.05 \mathrm{~mm}$

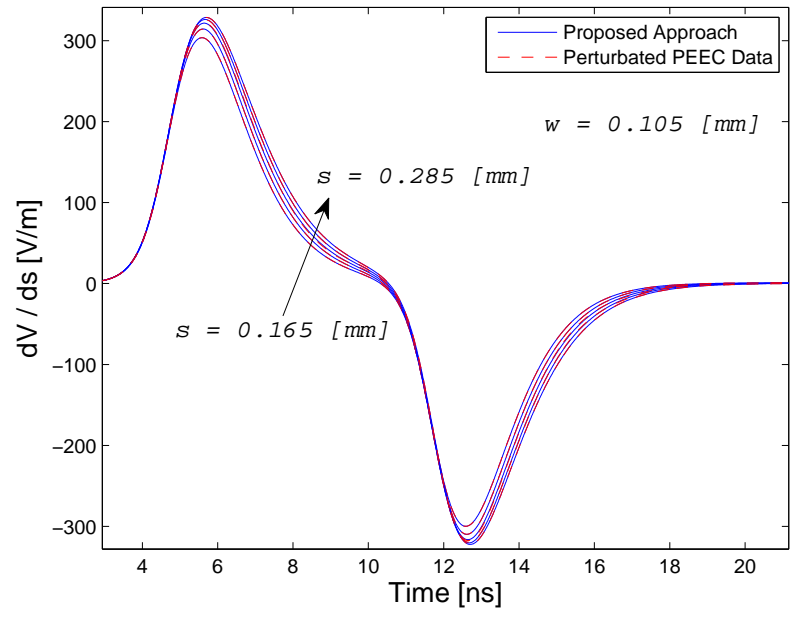

Fig. 6. Time-domain voltage sensitivity with respect to $s$ for the spiral inductor (Section VI-A).

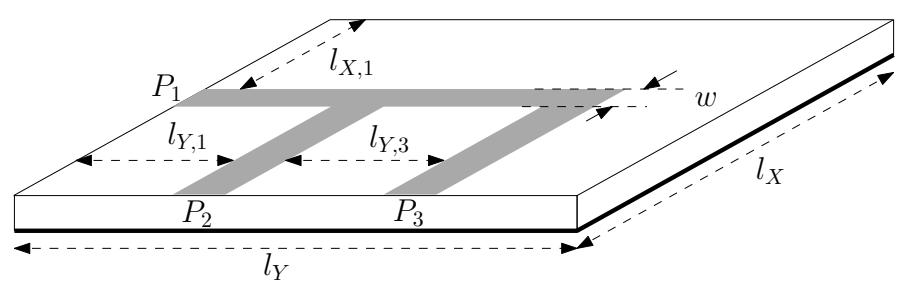

Fig. 7. Three-port microstrip power-divider circuit.

and the dielectric thickness $t_{\text {Diel }}=0.5 \mathrm{~mm}$. As in the previous example, a trivariate model has been built as a function of $l_{X, 1} \in[4-15] \mathrm{mm}$ and $w \in[0.8-1.2] \mathrm{mm}$, in addition to time. The interpolation process has been performed using a uniform sampling grid composed of $6 \times 6\left(l_{X 1}, w\right)$ values according to Section IV. Port $P_{1}$ has been excited by a smooth pulse voltage source with amplitude $1 \mathrm{~V}$, rise/fall times $\tau_{r}=$ $\tau_{f}=1.5 \mathrm{~ns}$ and width $3 \mathrm{~ns}$. Fig. 8 shows the ports voltages for $l_{X 1}=5.1 \mathrm{~mm}$ and $w=0.84 \mathrm{~mm}$, while Figs. 9 and 10 show the parameterized voltage sensitivity with respect to $l_{X 1}$ and $w$, respectively, for five values of $l_{X 1}$, equidistantly spaced between $5.1 \mathrm{~mm}$ and $13.9 \mathrm{~mm}$, and for five values of $w$, equidistantly spaced between $0.84 \mathrm{~mm}$ and $1.16 \mathrm{~mm}$. So, again, these values are chosen outside the sampling grid to allow a fair comparison. The numerical results confirm the high accuracy of the presented algorithm. Table III shows that the proposed model is almost twice faster than the perturbative approach, apart from the computational cost to generate the interpolation models, for which the same considerations made previously are valid.

\section{CONCLUSIONS}

This paper has presented a new method to perform parameterized sensitivity analyses of multiport systems that depend on multiple design parameters. It is based on the reliable and efficient combination of the PEEC method and suitable interpolation schemes, which are able to provide 
TABLE II

COMPUTATional Results (CPU TIME) FOR EXAMPLE VI-A

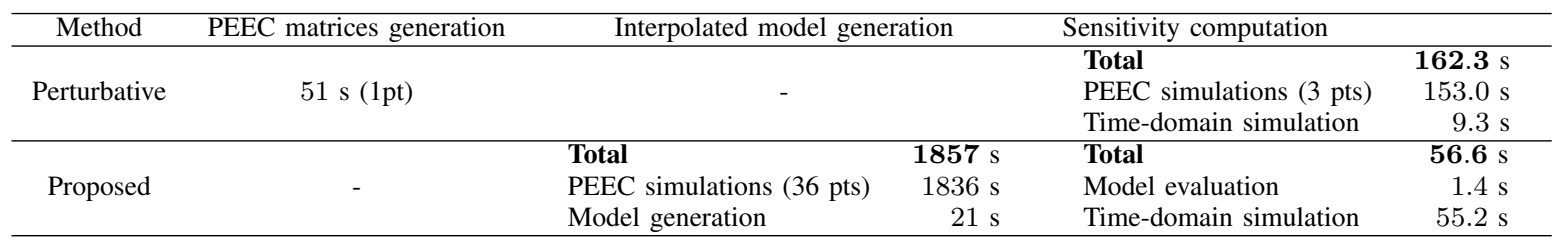

TABLE III

COMPutational Results (CPU TIME) FOR EXAMPLE VI-B

\begin{tabular}{|c|c|c|c|c|c|}
\hline Method & PEEC matrices generation & Interpolated model gen & ation & Sensitivity computation & \\
\hline Perturbative & $122 \mathrm{~s}(1 \mathrm{pt})$ & \multicolumn{2}{|l|}{ - } & Total & $397.8 \mathrm{~s}$ \\
\hline \multirow[t]{2}{*}{ Proposed } & \multirow[t]{2}{*}{ - } & PEEC simulation (36 pts) & $4392 \mathrm{~s}$ & Model evaluation & $5.4 \mathrm{~s}$ \\
\hline & & Model generation & $36 \mathrm{~s}$ & Time-domain simulation & $200.0 \mathrm{~s}$ \\
\hline
\end{tabular}

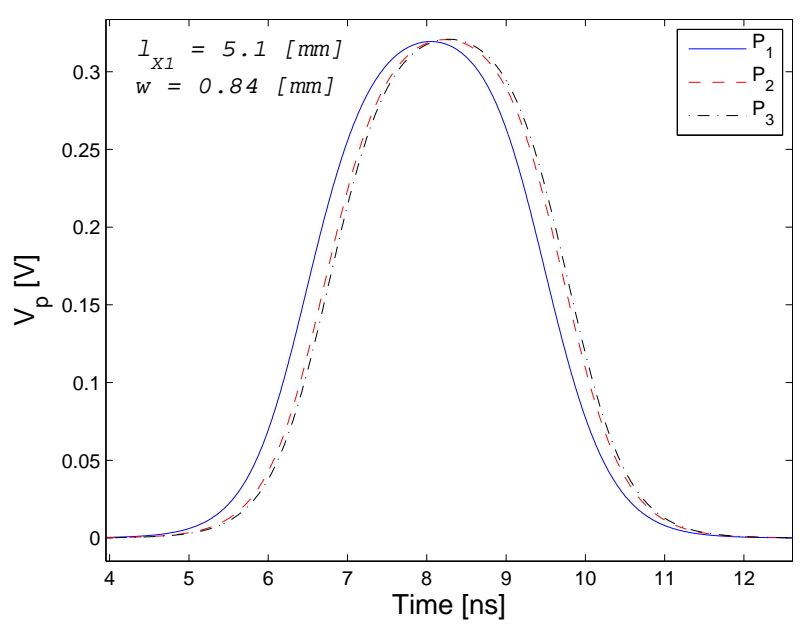

Fig. 8. Time-domain voltage at ports $P_{1}, P_{2}$ and $P_{3}$ of the power divider (Section VI-B).

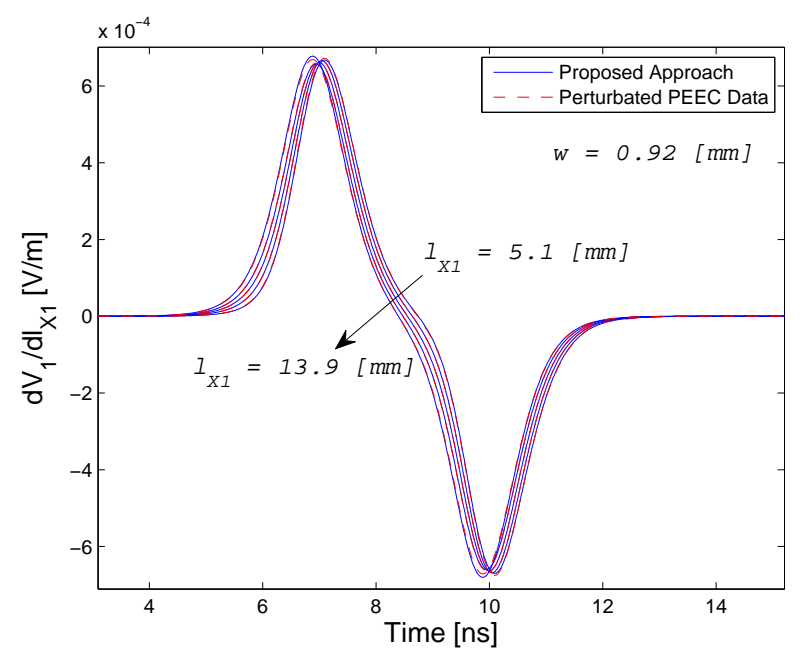

Fig. 9. Time-domain voltage sensitivity at port $P_{1}$ of the power divider with respect to $l_{X 1}$ (Section VI-B).

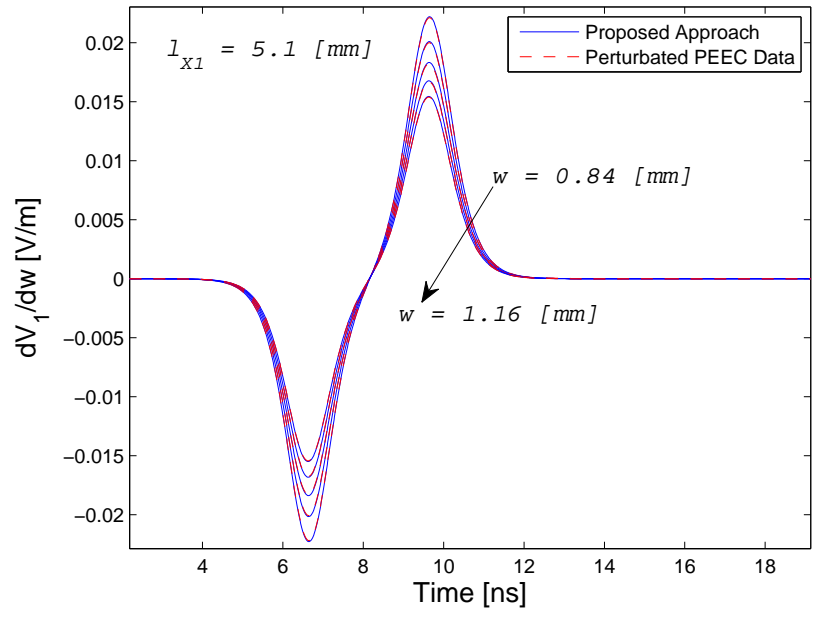

Fig. 10. Time-domain voltage sensitivity at port $P_{1}$ of the power divider with respect to $w$ (Section VI-B).

passive models over the entire design space along with the corresponding sensitivities. We have discussed the required stability and passivity properties of the systems involved in the parameterized sensitivity analysis to guarantee stable timedomain simulations. Pertinent numerical results have validated the proposed method and confirm its high modeling capability and efficiency with respect to the perturbative approach. Once the model has been generated, the system responses and corresponding sensitivities can be efficiently computed over the entire design space of interest. Instead, the perturbative approach needs additional simulations for each new point in the design space and demands for a careful choice of the perturbation values, which are often difficult to estimate.

\section{REFERENCES}

[1] E. A. Soliman, M. H. Bakr, and N. K. Nikolova, "An adjoint variable method for sensitivity calculations of multiport devices," IEEE Transactions on Microwave Theory and Techniques, vol. 52, no. 2, Feb. 2004.

[2] J.-F. Mao and E. S. Kuh, "Fast simulation and sensitivity analysis of lossy transmission lines by the method of characteristics," IEEE Transactions on Circuits and Systems, I, vol. 44, no. 5, May 1997. 
[3] A. E. Ruehli, "Equivalent circuit models for three dimensional multiconductor systems," IEEE Transactions on Microwave Theory and Techniques, vol. MTT-22, no. 3, pp. 216-221, Mar. 1974.

[4] C. A. Balanis, Advanced engineering electromagnetics. John Wiley and Sons, New York, 1989.

[5] A. E. Ruehli, A. C. Cangellaris, "Progress in the methodologies for the electrical modeling of interconnect and electronic packages," Proceedings of the IEEE, vol. 89, no. 5, pp. 740-771, May 2001.

[6] W. Pinello, A. C. Cangellaris and A. E. Ruehli, "Hybrid electromagnetic modeling of noise interactions in packaged electronics based on the partial-element equivalent circuit formulation," IEEE Transactions on Microwave Theory and Techniques, vol. MTT-45, no. 10, pp. 18891896, Oct. 1997.

[7] P. J. Restle, A. Ruehli, S. G. Walker, G. Papadopoulos, "Full-wave PEEC time-domain for the modeling of on-chip interconnects," IEEE Transactions on Computer-Aided Design, vol. 20, no. 7, pp. 877-887, Jul. 2001.

[8] G. Wollenberg, A. Görisch, "Analysis of 3-D interconnect structures with PEEC using SPICE," IEEE Transactions on Electromagnetic Compatibility, vol. 41, no. 2, pp. 412-417, Nov. 1999.

[9] G. Antonini, A. Orlandi, "A wavelet based time domain solution for PEEC circuits," IEEE Transactions on Circuits and Systems, vol. 47, no. 11 , pp. $1634-1639$, Nov. 2000.

[10] J. Cullum, A. Ruehli, T. Zhang, "A method for reduced-order modeling and simulation of large interconnect circuits and its application to PEEC models with retardation," IEEE Transactions on Circuits and Systems, II, vol. 47, no. 4, pp. 261-373, Apr. 2000.

[11] A. Ruehli, G. Antonini, J. Esch, J. Ekman, A. Mayo, A. Orlandi, "Nonorthogonal PEEC formulation for time and frequency domain EM and circuit modeling," IEEE Transactions on Electromagnetic Compatibility, vol. 45, no. 2, pp. 167-176, May 2003.

[12] L. W. Nagel, "SPICE: A computer program to simulate semiconductor circuits," University of California, Berkeley, Electr. Res. Lab. Report ERL M520, May 1975.

[13] F. Ferranti, G. Antonini, T. Dhaene, L. Knockaert, and A. Ruehli, "Physics-based passivity-preserving parameterized model order reduction for PEEC circuit analysis," IEEE Transactions on Components, Packaging and Manufacturing Technology, vol. 1, no. 3, pp. 399 - 409, Mar. 2011.

[14] C. Ho, A. Ruehli, P. Brennan, "The modified nodal approach to network analysis," IEEE Transactions on Circuits and Systems, pp. 504 - 509, Jun. 1975.

[15] F. Ferranti, G. Antonini, T. Dhaene, and L. Knockaert, "Guaranteed passive parameterized model order reduction of the partial element equivalent circuit (PEEC) method," IEEE Transactions on Electromagnetic Compatibility, vol. 52, no. 4, pp. 974 -984, Nov. 2010.

[16] A. E. Ruehli and H. Heeb, "Circuit models for three-dimensional geometries including dielectrics," IEEE Transactions on Microwave Theory and Techniques, vol. 40, no. 7, pp. 1507-1516, Jul. 1992.

[17] A. Odabasioglu, M. Celik, and L. Pileggi, "Prima: passive reducedorder interconnect macromodeling algorithm," IEEE Transactions on Computer-Aided Design of Integrated Circuits and Systems, vol. 17, no. 8, pp. 645 -654, Aug 1998.

[18] C. De Boor, A practical guide to splines. Springer-Verlag Berlin and Heidelberg GmbH \& Co. K, 1978.

[19] B. D. Anderson, S. Vongpanitlerd, Network Analysis and Synthesis. Englewood Cliffs, NJ, 1973.

[20] R. W. Freund, "Krylov-subspace methods for reduced-order modeling in circuit simulation," J. Comput. Appl. Math., vol. 123, no. 1-2, pp. 395 $-421,2000$

[21] R. Wang and J.-M. Jin, "A flexible time-stepping scheme for hybrid field-circuit simulation based on the extended time-domain finite element method," IEEE Transactions on Advanced Packaging, vol. 33, no. 4, pp. 769 - 776, Nov. 2010 